\title{
An Efficient Deep Learning Framework for Low Rate Massive MIMO CSI Reporting
}

\author{
Zhenyu Liu, Lin Zhang, and Zhi Ding
}

\begin{abstract}
Channel state information (CSI) reporting is important for multiple-input multiple-output (MIMO) transmitters to achieve high capacity and energy efficiency in frequency division duplex (FDD) mode. CSI reporting for massive MIMO systems could consume excessive bandwidth and degrade spectrum efficiency. Deep learning (DL)-based compression integrated with channel correlations have demonstrated success in improving CSI recovery. However, existing works focusing on CSI compression have shown little on the efficient encoding of CSI report. In this paper, we propose an efficient DL-based compression framework (called CQNet) to jointly tackle CSI compression, report encoding, and recovery under bandwidth constraint. CQNet can be directly integrated within other DL-based CSI feedback works for further enhancement. CQNet significantly outperforms solutions using uniform CSI quantization and $\mu$-law non-uniform quantization. Compared with traditional CSI reporting, much fewer bits are required to achieve comparable CSI reconstruction accuracy.
\end{abstract}

\section{Index Terms}

Massive MIMO, FDD, CSI feedback, quantization, deep learning.

\section{INTRODUCTION}

Massive multiple-input multiple-output (MIMO) systems have shown great promise in delivering high spectrum and energy efficiency for $5 \mathrm{G}$ and future wireless communication systems

Z. Liu is with the School of Information and Communication Engineering, Beijing University of Posts and Telecommunications, Beijing 100876, China, and also with the Department of Electrical and Computer Engineering, University of California at Davis, Davis, CA 95616 USA (e-mail: lzyu@bupt.edu.cn).

L. Zhang is with the School of Information and Communication Engineering, Beijing University of Posts and Telecommunications, Beijing 100876, China (e-mail: zhanglin@bupt.edu.cn).

Z. Ding is with the Department of Electrical and Computer Engineering, University of California at Davis, Davis, CA 95616 USA (e-mail: zding@ucdavis.edu). 
[1]. By utilizing a large number of antennas in massive MIMO framework, gNB (or gNodeB) in $5 \mathrm{G}$ can achieve very high downlink throughput if sufficiently accurate downlink channel state information (CSI) is available at the gNB. Consequently, gNB needs to acquire the downlink CSI in an accurate and timely manner to fully utilize the spatial diversity and multiplexing gains.

In time division duplex (TDD) systems, gNB can leverage its uplink CSI as the close estimate of its downlink CSI based on the well known reciprocity between downlink and uplink CSIs. In frequency division duplex (FDD) systems, however, uplink and downlink channels are in different frequency bands. Thus, it is difficult to only rely on uplink CSI to estimate the downlink CSI as the bi-directional channel reciprocity no longer applies. Consequently, gNB transmitters of FDD systems would require user equipment (UE) to provide certain CSI reporting about the downlink CSI. For massive MIMO, such feedback data can be substantial since the large number of antennas leads to very high CSI dimensionality. Large bandwidth in high rate links further exacerbates the high feedback load.

To improve spectrum efficiency for CSI reporting in FDD systems, compressed sensing (CS)based approaches can exploit the CSI properties of low rank or sparsity to derive a compressed CSI representation for feedback. Two major correlation properties used in CS-based CSI feedback approaches include spatial CSI correlation [2], [3], [4] that stems from the limited scattering characteristics of signal propagation, and temporal CSI correlation [5] owing to Doppler effects. However, CS-based approaches still have some limitations. On the one hand, CS-based approaches require a strong channel sparsity condition which is not strictly held in some cases. On the other hand, CS algorithms are often iterative and computationally intensive during decoding procession, which may lead to long delays.

Deep learning (DL) is a powerful tool for exploring the underlying structures from large data set, and has been widely used in computer vision and natural language processing. It can play a helpful role in CSI estimation when traditional methods generate limited performance. There have been some successful applications to derive reliable downlink CSI in massive MIMO systems for channel estimation [6] and low rate CSI feedback [7], [8]. In particular for massive MIMO, the authors of [7] developed a CSI compression and recovery mechanism using an autoencoder structure [9], and demonstrated better accuracy than CS-based methods in terms of downlink CSI reconstruction from limited UE feedback. The work of [8] further exploited the FDD bi-directional channel correlation. By jointly utilizing the available uplink CSI and low rate UE feedback in massive MIMO systems at $\mathrm{gNB}$ to recover the unknown downlink CSI, 
significant performance gain of downlink CSI estimate was shown over DL architecture based only on UE feedback [8].

Common implementation of DL networks complies with IEEE 754 standard [10]. Single precision, which is defined in this standard and used in [7], [8], is the most typical data type adopted in DL networks [11]. Although DL methods have delivered noticeable performance improvement in reducing the dimension of CSI matrices in massive MIMO, current CSI reporting results that use single precision (32-bit) to encode feedback coefficients still consume too much bandwidth. To reduce the number of bits required by each codeword, low-bit quantization should take place in encoding after dimension compression. Clearly, low-bit quantization for CSI codewords will degrade CSI reconstruction accuracy. Therefore, it is important to jointly optimize the encoding and dimension compression to maintain high CSI reconstruction accuracy without consuming excessive bandwidth in FDD systems.

In this work, we propose an efficient CSI compression solution and design an end-to-end DL framework CQNet to optimize downlink CSI compression and feedback encoding simultaneously. Our CQNet is a simple plug-in that can be integrated into existing DL-based CSI feedback frameworks. We also investigate how feedback bandwidth affects CSI reconstruction accuracy under a uniform quantization framework. Moreover, we use two existing works CsiNet [7] and DualNet [8] as examples to show how to implement the CQNet to improve bandwidth efficiency. To enhance the flexibility of phase quantization in DualNet, we also propose a DLbased magnitude-adaptive phase encoder, which can easily adjust quantization bits according to required accuracy.

Our contributions in this paper are summarized as follows:

- We evaluate how CSI reconstruction accuracy is affected by the feedback bandwidth under uniform quantization, and show significant bandwidth savings over single precision feedback of compressed CSI coefficients.

- We develop a CQNet that can simultaneously optimize the dimension compression and encoding of downlink CSI to improve the bandwidth efficiency. The CQNet can be directly combined with existing DL-based CSI feedback methods to save bandwidth.

- We analyze different encoding and quantization codewords to demonstrate the advantages of CQNet.

- We further design a specialized DL-based phase quantization framework that can achieve the magnitude-adaptive phase quantization. This framework increases the flexibility of phase 
quantization and can regulate the weight of quantization entropy to achieve a balanced bandwidth versus CSI accuracy design trade-off.

- Test results demonstrate that CQNet significantly outperforms uniform quantization and $\mu$ law quantization. Compared with single-precision feedback, CQNet can achieve comparable CSI reconstruction accuracy using 5 bits per coefficient in the feedback. CQNet with entropy encoding can further reduce down to 4 bits per coefficient.

\section{RELATED WORKS}

FDD base stations like eNB or gNB typically rely on CSI feedbacks to report downlink CSI due to the weak reciprocity between uplink and downlink channels. To implement massive MIMO downlink in FDD, feedback payload is substantially high because of the large antenna number and wide bandwidth. Conventional methods based purely on UE feedback face several challenges including channel models and feedback bandwidth consumption. Recognizing the importance to conserve feedback bandwidth and improve downlink CSI reconstruction accuracy, there has been a recent surge of interest in DL-based CSI feedback [7], [8], [12], [13].

Among related works, a DL-based CSI feedback framework CsiNet for massive MIMO downlink was proposed in [7] to reduce UE feedback overhead. As shown in Fig. 1 (a), CsiNet utilizes an autoencoder architecture, where an encoder deep neural network (DNN) acts as a compression module and a corresponding decoder DNN is responsible for CSI reconstruction. Treating CSI matrix as a virtual image, convolutional layer is used in both encoder and decoder to exploit its spatial and spectral correlation. Fully connected layers are used for dimension compression and decompression. Each CSI matrix is split into real and imaginary parts, rearranged into two sets of encoder DNN input. CsiNet demonstrated performance gain over CS-based methods.

Another DL-based CSI feedback framework DualNet-MAG established the bi-directional correlation among the magnitudes of FDD channel coefficients [8]. DualNet-MAG demonstrated significant performance benefit of exploiting this uplink-downlink CSI correlation in reducing the amount of CSI feedback [8]. Fig. 1 (b) shows that, unlike CsiNet, DualNet exploits the available uplink CSI at gNB to help recover the downlink CSI from lower rate CSI feedback. The bidirectional channel correlation between CSI magnitudes helps the decoder DNN recover the downlink CSI magnitudes with better accuracy by leveraging the low-rate feedback codewords and locally available uplink CSI magnitudes. The work in [8] also shows that the CSI phase correlation is weak between uplink and downlink CSIs. Thus, CSI feedback should focus on 


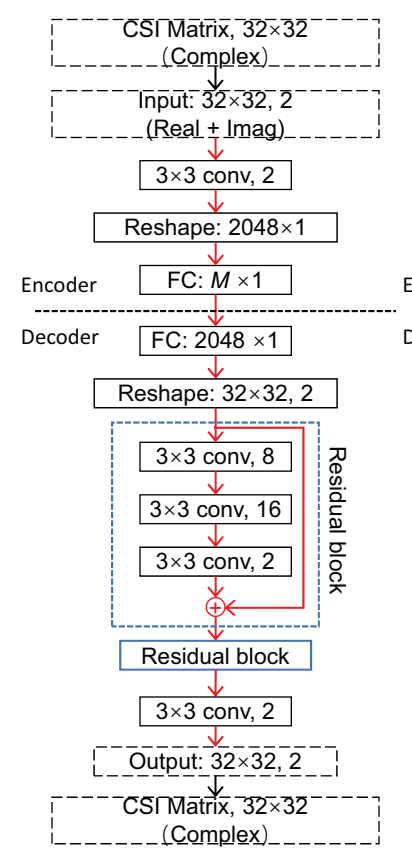

(a)

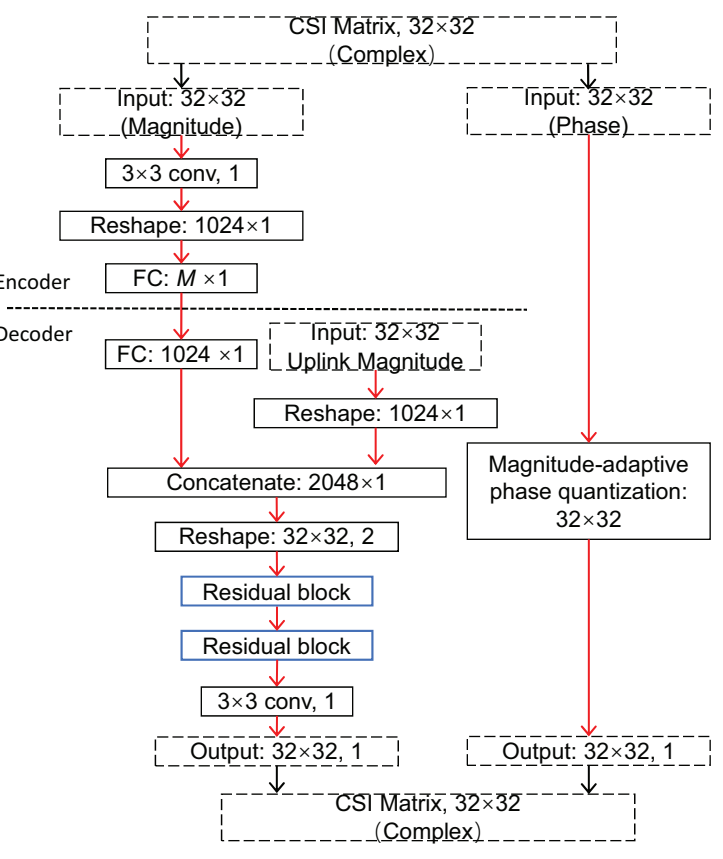

(b)

Fig. 1: Architecture of CsiNet and DualNet-MAG. CsiNet consists of an encoder network with a 2-channel $3 \times 3$ convolutional (conv) layer and an $M \times 1$ fully connected layer (FC) for dimension compression and a decoder using a FC for dimension decompression and two Residual blocks for CSI calibration. DualNet-MAG concatenates the feedback codewords for magnitude with uplink CSI's magnitude before feeding into the residual blocks. The phase of CSI is fed back using the magnitude-adaptive quantization.

CSI phase encoding and a magnitude-adaptive phase encoding can reduce the phase feedback overhead and achieve clear performance improvement over traditional methods and CsiNet.

Another work [12] proposed the use of long-short time memory (LSTM) network [14] known as CsiNet-LSTM to exploit the temporal correlation of CSI. In [13], a new LSTM network further reduced the number of parameters to be trained while maintaining the CSI recovery accuracy.

However, the aforementioned works on DL-based CSI feedback generally focused on dimension reduction of CSI feedback. Single precision or float32 (defined in IEEE 754 standard) is the most typical data type adopted in DL networks [11] and has been widely used in channel estimation [6] and channel feedback [7], [8]. Although single precision can help DL networks gain great accuracy, bandwidth efficient encoding of dimension-reduced CSI coefficients while maintaining the comparable accuracy remains a challenge. 
A recent work [15] showed that encoding precision can be reduced with manageable accuracy loss in image classification. To achieve better CSI reporting efficiency, we investigate the encoding of compressed CSI coefficients for limited feedback bandwidth with minimum loss of CSI reconstruction accuracy. In this paper, we first evaluate the impact of feedback bandwidth on CSI reconstruction accuracy under uniform quantization/encoding, and show that quantization can significantly save the bandwidth. Based on this observation, we further propose a novel learning framework to jointly optimize the dimension compression and CSI encoding at the same time. This framework can reduce the bandwidth required by downlink CSI feedback while preserving high CSI reconstruction accuracy. It can also be easily integrated with the existing DL-based CSI feedback works.

There are a few other recent works involved the quantization of the CSI codewords. In [16], the $\mu$-law quantization was utilized to encode the codewords after dimension compression. However, neither $\mu$-law quantization nor uniform quantization alone can efficiently encode CSI feedbacks, which we shall demonstrate in the performance evaluation section of this work. Another recent method [17] proposed a specific quantization DL network with successful results. Advancing further, our framework in this work can customize quantization parameters for each CSI codeword and can be easily integrated into existing CSI feedback works without additional modifications.

\section{SySTEM MODEL}

Consider a single-cell massive MIMO system, in which the gNB has $N_{b} \gg 1$ antennas and UEs have a single antenna. The system applies orthogonal frequency division multiplexing (OFDM) over $N_{f}$ subcarriers, for which the downlink received signal at the $n$-th subcarrier is

$$
y_{d}^{(n)}=\mathbf{h}_{d}^{(n)}{ }^{H} \mathbf{w}_{T}^{(n)} x_{d}^{(n)}+n_{d}^{(n)},
$$

where $\mathbf{h}_{d}^{(n)} \in \mathbb{C}^{N_{b} \times 1}$ denotes the channel vector of the $n$-th subcarrier, $\mathbf{w}_{T}^{(n)} \in \mathbb{C}^{N_{b} \times 1}$ denotes transmit beamformer, $x_{d}^{(n)} \in \mathbb{C}$ is the transmitted symbol, and $n_{d}^{(n)} \in \mathbb{C}$ denotes the additive noise. $(\cdot)^{H}$ denotes conjugate transpose. With the downlink channel vector $\mathbf{h}_{d}^{(n)}$, gNB can calculate the transmit beamformer $\mathbf{w}_{T}^{(n)}$. The uplink received signal of the $n$-th subcarrier is given by

$$
y_{u}^{(n)}=\mathbf{w}_{R}^{(n)}{ }^{H} \mathbf{h}_{u}^{(n)} x_{u}^{(n)}+\mathbf{w}_{R}^{(n)}{ }^{H} \mathbf{n}_{u}^{(n)},
$$

where $\mathbf{w}_{R}^{(n)} \in \mathbb{C}^{N_{b} \times 1}$ denotes the receive beamformer, and subscript $u$ denotes uplink signals and noise, similar to (1). The downlink and uplink CSI matrices in the spatial frequency domain 


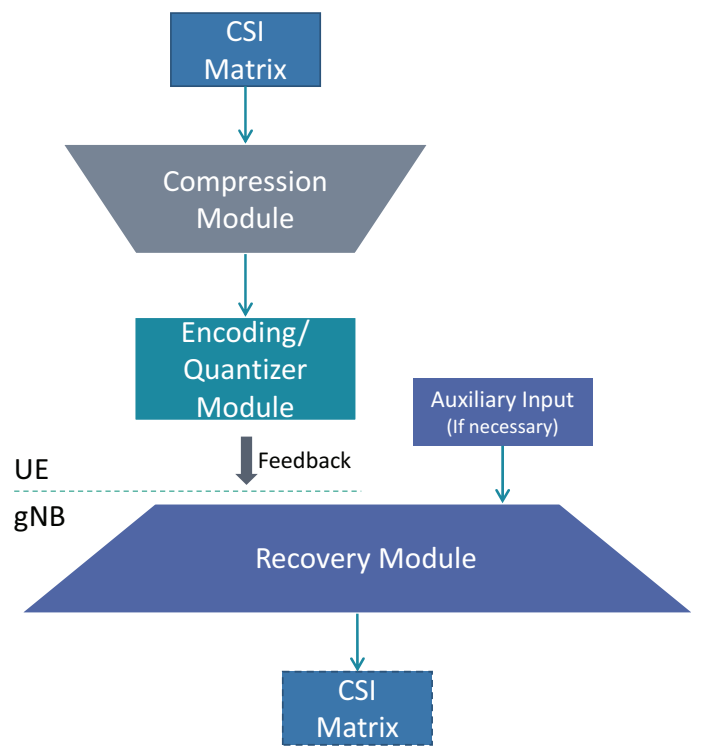

Fig. 2: CSI feedback framework.

are denoted as $\tilde{\mathbf{H}}_{d}=\left[\mathbf{h}_{d}^{(1)}, \ldots, \mathbf{h}_{d}^{\left(N_{f}\right)}\right]^{H} \in \mathbb{C}^{N_{f} \times N_{b}}$ and $\tilde{\mathbf{H}}_{u}=\left[\mathbf{h}_{u}^{(1)}, \ldots, \mathbf{h}_{u}^{\left(N_{f}\right)}\right]^{H} \in \mathbb{C}^{N_{f} \times N_{b}}$, respectively.

To reduce the feedback overhead, we first exploit the property that CSI matrices exhibit some sparsity in the delay domain since the delay among multiple paths lies in a particularly limited period [18]. The CSI matrix $\mathbf{H}_{f}$ in frequency domain can be transformed to be $\mathbf{H}_{t}$ in delay domain using an inverse discrete Fourier transform (IDFT), i.e.,

$$
\mathbf{H}_{f} \mathbf{F}^{H}=\mathbf{H}_{t},
$$

where $\mathbf{F}$ and $\mathbf{F}^{H}$ denote the $N_{f} \times N_{f}$ unitary DFT matrix and IDFT matrix, respectively. After IDFT of (3), most elements in the $N_{f} \times N_{b}$ matrix $\mathbf{H}_{t}$ are near zero except for the first $Q_{f}$ rows. Therefore, we truncate the channel matrix to the first $Q_{f}$ rows that are with distinct non-zero values, and utilize $\mathbf{H}_{d}$ and $\mathbf{H}_{u}$ to denote the first $Q_{f}$ rows of matrices after IDFT of $\tilde{\mathbf{H}}_{d}$ and $\tilde{\mathbf{H}}_{u}$, respectively.

To reduce the redundancy in reporting downlink CSI, the codewords should be dimensioncompressed and low-bit encoded. Consequently, unlike CsiNet and DualNet-MAG which only utilize the encoder and decoder for CSI dimension compression and reconstruction respectively, a quantizer module is added between the encoder and decoder to execute dimension compression and encoding/quantization jointly. 
As shown in Fig. 2, our CSI feedback framework for FDD downlink channel reconstruction consists of 3 modules: a compression module, an encoding/quantizer module, and a recovery module. Specifically, we design a DNN architecture CQNet to jointly optimize the dimension compression and encoding process at the receiver. The recovery module at the transmitter is correspondingly optimized for CSI recovery. We shall illustrate that the CQNet framework can be integrated with existing dimension compression methods such as CsiNet in [7] and DualNetMAG in [8]. Jointly optimizing compression and encoding of downlink CSI feedback, these corresponding new architectures are respectively named as CsiQnet and DualQnet.

We shall let $\hat{\mathbf{H}}_{d}$ denote the reconstructed downlink CSI matrix. We define the encoding/quantization function as $f_{\text {quan }}(\cdot)$. For CsiQnet, the dimension compression module, quantizer module, decoder module can be denoted, respectively, by

$$
\begin{aligned}
\mathbf{s}_{1} & =f_{c, 1}\left(\mathbf{H}_{d}\right), \\
\hat{\mathbf{s}}_{1} & =f_{\text {quan }, 1}\left(\mathbf{s}_{1}\right), \\
\hat{\mathbf{H}}_{d} & =f_{r, 1}\left(\hat{\mathbf{s}}_{1}\right) .
\end{aligned}
$$

For DualQnet, the dimension compression module, quantizer module, decoder module can be denoted, respectively, by

$$
\begin{aligned}
\mathbf{s}_{2} & =f_{c, 2}\left(\mathbf{H}_{d}\right), \\
\hat{\mathbf{s}}_{2} & =f_{\text {quan }, 2}\left(\mathbf{s}_{2}\right), \\
\hat{\mathbf{H}}_{d} & =f_{r, 2}\left(\hat{\mathbf{s}}_{2}, \mathbf{H}_{u}\right) .
\end{aligned}
$$

The optimization of downlink CSI recovery method can be formulated as minimizing $\left\|\mathbf{H}_{d}-\hat{\mathbf{H}}_{d}\right\|^{2}$, where $\|\cdot\|$ is Frobenius norm.

\section{BANDLIMITED CSI FEEDBACK}

The DL-based CSI feedback works including CsiNet in [7] and DualNet in [8] have demonstrated substantial performance gain in terms of downlink CSI feedback reduction and reconstruction accuracy. However, in addition to the benefit of downlink CSI compression, additional encoding of the compressed CSI feedback coefficients from the original float32 format [7], [8] can further reduce the downlink CSI feedback for massive MIMO systems drastically.

Toward this goal, we first evaluate the impact of quantization/encoding codeword length on CSI reconstruction accuracy by examining a simple uniform quantizer. We start by testing CsiNet 
and DualNet by simply adding the uniform quantizer between the encoder DNN and decoder DNN of CsiNet and DualNet to assess the impact of quantization distortion.

For CsiNet, Fig. 1 (a) shows the encoder network containing a $3 \times 3$ convolutional layer with 2 channels plus an $M$-unit fully connected layer for dimension compression. The decoder network consists of a fully connected layer for decompression and two residual blocks to reconstruct the downlink CSI. Each residual block contains three $3 \times 3$ convolutional layers with the channel number 8,16 , and 2, respectively.

DualNet-MAG leverages the magnitude correlation between uplink and downlink to improve CSI feedback efficiency. As shown in Fig. 11 (b), DualNet-MAG processes the magnitude and phase separately. After separation, the CSI magnitudes are sent to the encoder network including a $3 \times 3$ convolutional layer and an $M$-unit fully connected layer. The gNB decoder receives the compressed codewords and uses the locally available uplink CSI magnitudes together to jointly decode downlink CSI. The received codewords are first mapped to their original length using a fully connected layer. The conjugation layer combines both downlink CSI and uplink CSI to generate an output reshaped into 2 feature maps to be used as input to the residual blocks. To save feedback bandwidth while limiting quantization error, a magnitude-adaptive phase quantization (MAPQ) is applied in which CSI coefficients with larger magnitude receive finer phase quantization, and vice versa.

Uniform quantization is simple and well known in practice. It is basically a rounding process, in which each sample value is rounded to the nearest value among a finite set of possible quantization levels. We can normalize to limit the amplitude of CSI coeffients between $\left[s_{\min }, s_{\max }\right]$. Let $\ell$ be the number of bits for amplitude quantization. Each CSI coefficient's amplitude can be uniformly quantized into $2^{\ell}$ levels:

$$
\hat{s}=\Delta\left\lfloor\frac{s}{\Delta}\right\rceil, \quad \text { where } \quad \Delta=\frac{s_{\max }-s_{\min }}{2^{\ell}-1} .
$$

We include uniform quantization into the DL-based CSI feedback framework. Specifically, we first train CsiNet and DualNet-MAG without quantization in the original end-to-end approach. Next, we use uniform quantization to digitize the compressed CSI coefficients, before sending the quantized CSI values into the decoder.

In the next experiment, we use the COST 2100 channel model to generate CSI matrices for reconstruction evaluation [19]. A uniform linear array (ULA) of transmit antennas is set up with half-wavelength spacing in an indoor environment with uplink and downlink bands at $5.1 \mathrm{GHz}$ 


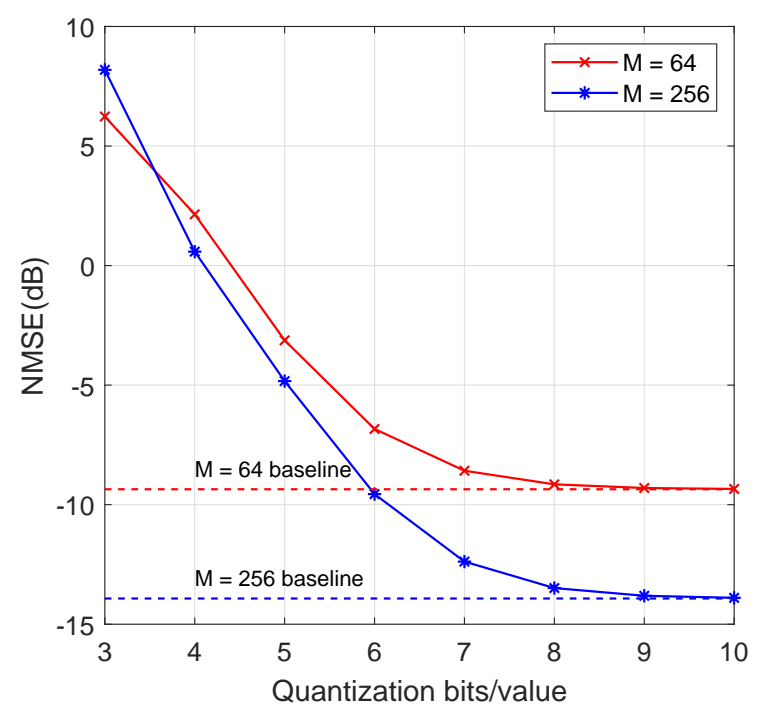

(a) CsiNet

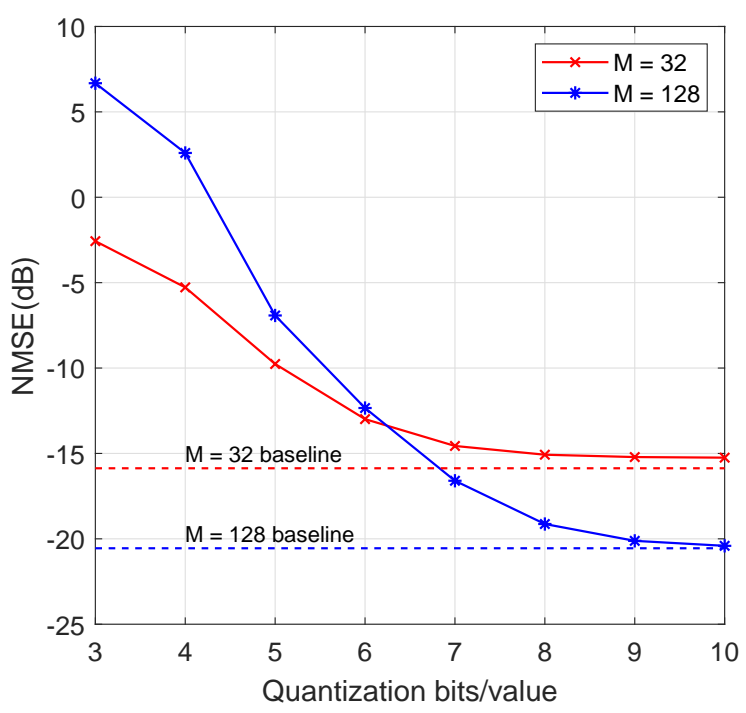

(b) DualNet-MAG

Fig. 3: CSI recovery accuracy at different quantization levels.

and $5.3 \mathrm{GHz}$, respectively. Fig. 3 shows the resulting NMSE for CsiNet and DualNet-MAG under different levels of quantization $2^{\ell}$ as we vary $\ell$ from 3 to 10 bits. For CsiNet, we set the length of compressed codeword vector to $M=64$ and 256, respectively. For DualNet-MAG, we set the length of compressed codeword vector to $M=32$ and 128, respectively. Results from float32 serve as the baseline in CSI feedback.

A few observations can be made from the results of Fig. 3. First, high precision feedback of 32 bits per CSI value is unnecessary, as 10-bit uniform quantizer achieves nearly the same accuracy as float32 for both CsiNet and DualNet. Second, both CsiNet and DualNet are more robust to quantization errors at lower compression. Third, DualNet generally achieves better accuracy. Finally, CSI reconstruction accuracy degrades with coarser quantization. When $\ell$ drops below 7 , the reconstruction experiences a clear degradation.

These test results motivate our study to design a more efficient and suitable encoding/quantization solution which can deliver high CSI reconstruction accuracy while using smaller $\ell$ to further improve the feedback bandwidth efficiency.

\section{CQNET}

Optimum encoding or quantization depends on the distribution of data under quantization and the performance metric. For example, non-uniform quantizer such as the $\mu$-law method improved 


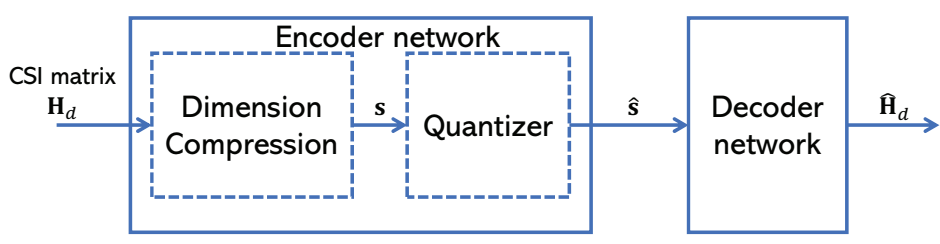

Fig. 4: Proposed CSI feedback framework CQNet.

the signal-to-quantization noise ratio (SQNR) for lower power signals. Clearly, it is impractical to exhaustively test a huge number of encoding schemes for the downlink CSI coefficients generated by the encoder DNN in order to determine the best fit. Instead, we shall develop a novel DNN approach to learn and optimize the quantization intervals in order to improve the CSI recovery accuracy for limited number of quantization levels.

\section{A. Joint Compression and Quantization Encoding}

We propose an end-to-end "CQNet" to jointly optimize CSI dimension reduction, encoding, and CSI reconstruction. As shown in Fig. 4, CQNet consists of an encoder DNN at the UE which includes a dimension compression module and a quantizer, paired with a decoder network at the gNB. The compression module can adopt the encoder neural network of CsiNet or DualNet. The quantizer module is parameterized by a trainable forward quantization weight vector $\mathbf{w}$ to map the unquantized $\mathbf{s}_{1}$ or $\mathbf{s}_{2}$, respectively, into an index vector $\mathbf{k}$ that corresponds to the quantized codeword $\hat{\mathbf{s}}_{1}$ or $\hat{\mathbf{s}}_{2}$. An inverse quantization weight vector $\mathbf{v}$ that generates approximate codeword $\hat{\mathbf{s}}_{i}$ from the index vector $\mathbf{k}$. To facilitate back-propagation during training, a soft quantizer is used to replace the non-differentiable quantizer function. After quantization, the jointly trained decoder DNN at the receiver is utilized to decode the quantized vector and reconstruct the downlink CSI matrix $\hat{\mathbf{H}}_{d}$.

In this framework, in addition to CSI dimension compression, we also optimize weight vectors $\left\{w_{i}\right\}$ within the quantization module which is part of the overall DNN. We define the weights of the quantizer such at the quantization intervals are $d_{i}=1 / w_{i}$ for each element $s_{i}$ in the compressed CSI vector s. As illustrated in Fig. 5, the forward quantization stage can be implemented as a set of element-wise multiplication filters which have only $M$ parameters followed by a rounding function. The rounding function can be viewed as an activation function of a neuron acting on each output from the element-wise multiplications. Since the rounding function Rnd 


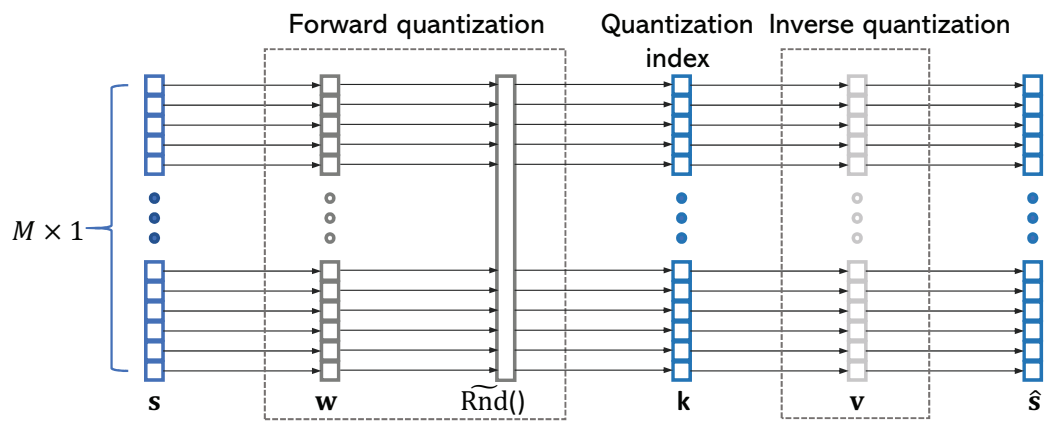

Fig. 5: Illustration of quantizer module. The quantizer has two distinct stages: forward quantization stage and inverse quantization stage. The forward stage maps the input vector $\mathbf{s}_{i}$ to an integer quantization index vector $\mathrm{k}$ and the reconstruction stage maps the index vector $\mathrm{k}$ back to vector $\hat{\mathbf{s}}_{i}$ as the approximation of the input vector.

has zero gradient almost everywhere, slow convergence may take place in back-propagation training. To overcome this shortcoming, we propose an approximate rounding function

$$
\widetilde{\operatorname{Rnd}}(x, \ell, r)=\sum_{i=-2^{\ell-1}}^{2^{\ell-1}-1} \operatorname{sigmoid}[r(x-i-0.5)]-2^{\ell-1},
$$

which is differentiable and easy to implement. $\widetilde{\operatorname{Rnd}}(\cdot)$ is a summation of sigmoid functions with parameters $\ell$ and $r$ where $\ell$ is the number of quantization bits for each element in CSI vector $\mathbf{s}$, and $r$ controls the sigmoidal shape. After forward quantization stage, an inverse quantization stage is added to reconstruct the approximated codeword $\hat{\mathbf{s}}_{i}$ using a vector $\mathbf{v}$ before feeding into the decoder module, as shown in Fig. 5

The CQNet framework is compatible with both CsiNet and DualNet-MAG, or any other CSI compression encoder. The CQNet architecture that uses CsiNet for CSI dimension reduction module and CSI recovery is named CsiQnet. The dimension compression module is followed by the quantizer module shown in Fig. 5. The decoder network of CsiNet is directly used in CsiQnet to decode the quantized codewords. The CQNet architecture that uses DualNet-MAG for CSI dimension reduction module and CSI recovery is named DualQnet. DualQnet uses the DualNet-MAG encoder network for dimension compression at the UE, and also uses the DualNet-MAG decoder network at the gNB to recover the CSI. Quantizer module is inserted between the dimension compression module and decoder module. 
We define the loss function of CsiQnet or DualQnet:

$$
L\left(\hat{\mathbf{H}}_{d}, \mathbf{w}\right)=L_{m}\left(\hat{\mathbf{H}}_{d}, \mathbf{H}_{d}\right)+\lambda L_{\text {quan }}(\mathbf{w})
$$

as a combination of mean square error (MSE) loss $L_{m}$ and a quantization loss $L_{\text {quan }}$. $L_{\text {quan }}$ is used for the regularization that accounts for quantization efficiency and convergency. One simple function for this purpose is $L_{\text {quan }}(\mathbf{w})=\|\mathbf{w}\|$. The training objective is to find the encoding and decoding parameters which can achieve the optimum CSI reconstruction accuracy given the specific quantization bits per value $\ell$.

Through training based on a large MIMO CSI data set generated using well known practical channel models such as COST 2100 model [19], CsiQnet and DualQnet can converge to optimized settings. During live downlink CSI feedback, both CsiQnet and DualQnet can generate more efficiently quantized codewords $\hat{\mathbf{s}}_{i}$ which can significantly improve the accuracy of CSI reconstruction at fixed bandwidth or bitwidth. CsiQnet and DualQnet enable more effective bandwidth usage with little CSI reconstruction loss.

To train the model, normalization is applied in both downlink and uplink CSI matrices. Adam optimizer is adopted to update the DL network parameters. The initial learning rate is set to 0.001. To accelerate the convergence speed of the training, we utilize the weights trained in CsiNet and DualNet-MAG to initialize the dimension compression modules and decoder networks of CsiQnet and DualQnet, respectively. Notice that DualQnet optimizes the magnitude feedback during joint training to minimize (12). For the separate phase feedback of compressed CSI, we shall design another MAPQ DNN in Section V-B.

\section{B. DL-based Phase Quantization}

DualNet-MAG utilizes the magnitude correlation of bi-directional CSIs in polar coordinate to reduce the amount of feedback for CSI magnitudes and improve the CSI feedback efficiency. However, weak phase correlation between uplink/downlink CSI requires the UE to efficiently quantize and encode all downlink CSI phases for feedback. However, it is well known that uniform phase quantization results in unnecessarily fine quantization at low magnitude and coarse quantization at high magnitude. Therefore, bandwidth efficiency phase quantization is an important issue to tackle in DualQnet.

Let $\mathbf{M}=\left[\mathbf{M}_{i, j}\right]$ be the magnitudes of CSI matrix, $\mathbf{P}=\left[\mathbf{P}_{i, j}\right]$ be the phases of CSI matrix, $\hat{\mathbf{M}}$ be the recovered magnitudes of CSI matrix, and $\hat{\mathbf{P}}$ be the recovered phases of CSI matrix, 
respectively. We can write a matrix $e^{j \mathbf{P}}$ whose elements are $e^{\mathbf{P}_{i, j}}$. The optimization objective of DualNet-MAG is to minimize the MSE of recovered CSI matrices, i.e., $\mathbf{E}\left\{\left\|\mathbf{H}_{d}-\hat{\mathbf{H}}_{d}\right\|^{2}\right\}=$ $\mathbf{E}\left\{\left\|\mathbf{M} \odot e^{j \mathbf{P}}-\hat{\mathbf{M}} \odot e^{j \hat{\mathbf{P}}}\right\|^{2}\right\}$, where $\odot$ denotes Hadamard matrix product. It is challenging to solve this problem since the recovered magnitude and phase influence the MSE jointly. To reduce the complexity of this problem, we consider the upper bound:

$$
\begin{aligned}
& \left\|\mathbf{M} \odot e^{j \mathbf{P}}-\hat{\mathbf{M}} \odot e^{j \hat{\mathbf{P}}}\right\|^{2} \\
& =\left\|\mathbf{M} \odot e^{j \mathbf{P}}-\mathbf{M} \odot e^{j \hat{\mathbf{P}}}+\mathbf{M} \odot e^{j \hat{\mathbf{P}}}-\hat{\mathbf{M}} \odot e^{j \hat{\mathbf{P}}}\right\|^{2} \\
& \leqslant 2\left\|\mathbf{M} \odot e^{j \mathbf{P}}-\mathbf{M} \odot e^{j \hat{\mathbf{P}}}\right\|^{2}+2\left\|\mathbf{M} \odot e^{j \hat{\mathbf{P}}}-\hat{\mathbf{M}} \odot e^{j \hat{\mathbf{P}}}\right\|^{2} \\
& =2\left\|\mathbf{M} \odot\left(e^{j \mathbf{P}}-e^{j \hat{\mathbf{P}}}\right)\right\|^{2}+2\|\mathbf{M}-\hat{\mathbf{M}}\|^{2} \cdot
\end{aligned}
$$

Consequently, the optimization goal can be relaxed to minimize

$$
\mathbf{E}_{(\mathbf{M}, \mathbf{P})}\left(\left\|\mathbf{M} \odot\left(e^{j \mathbf{P}}-e^{j \hat{\mathbf{P}}}\right)\right\|^{2}\right)+\mathbf{E}_{\mathbf{M}}\left(\|\mathbf{M}-\hat{\mathbf{M}}\|^{2}\right) .
$$

DualNet can minimize $\mathbf{E}_{\mathbf{M}}\left(\|\mathbf{M}-\hat{\mathbf{M}}\|^{2}\right)$. It is clear that the first part $\mathbf{E}_{(\mathbf{M}, \mathbf{P})}\left(\left\|\mathbf{M} \odot\left(e^{j \mathbf{P}}-e^{j \hat{\mathbf{P}}}\right)\right\|^{2}\right)$ represents phase quantization error amplified by the corresponding magnitude.

Therefore, to further reduce feedback bandwidth, CSI quantization error can be kept small by applying the magnitude-adaptive phase quantization (MAPQ) principle in which CSI coefficients with larger magnitude adopt finer phase quantization, and vice versa. After recovering the magnitude, gNB can restore the quantified phase based on MAPQ. Such MAPQ can keep the quantization error close for a range of magnitudes. Since quantization bits of phase vary with the magnitude, the expectation of quantization bits depends on the distribution of CSI magnitude. Thus, we need to allocate the the number of phase quantization bits based on the distribution of CSI magnitude underlimited average bitwidth. Such problems typically become a mixed integer nonlinear programming problem as described in [20], which is NP-hard.

A heuristic quantization bit allocation solution was provided in [8] based on examining the data set. In this method, the cumulative distribution function (CDF) of CSI magnitudes is estimated to determine magnitude values corresponding to $\mathrm{CDF}$ value of $0.5,0.7,0.8$, and 0.9 , respectively. These four points divide the CSI magnitudes into five ordered segments from low to high. Accordingly, 3, 4, 5, 6, 7 phase quantization bits are allocated, respectively, to encode the CSI phases. This set of MAPQ codewords given in [8] can generate codeword of the mean length of 4.1 bits to achieve the same MSE as that obtained using a 6-bit uniform quantizer. 


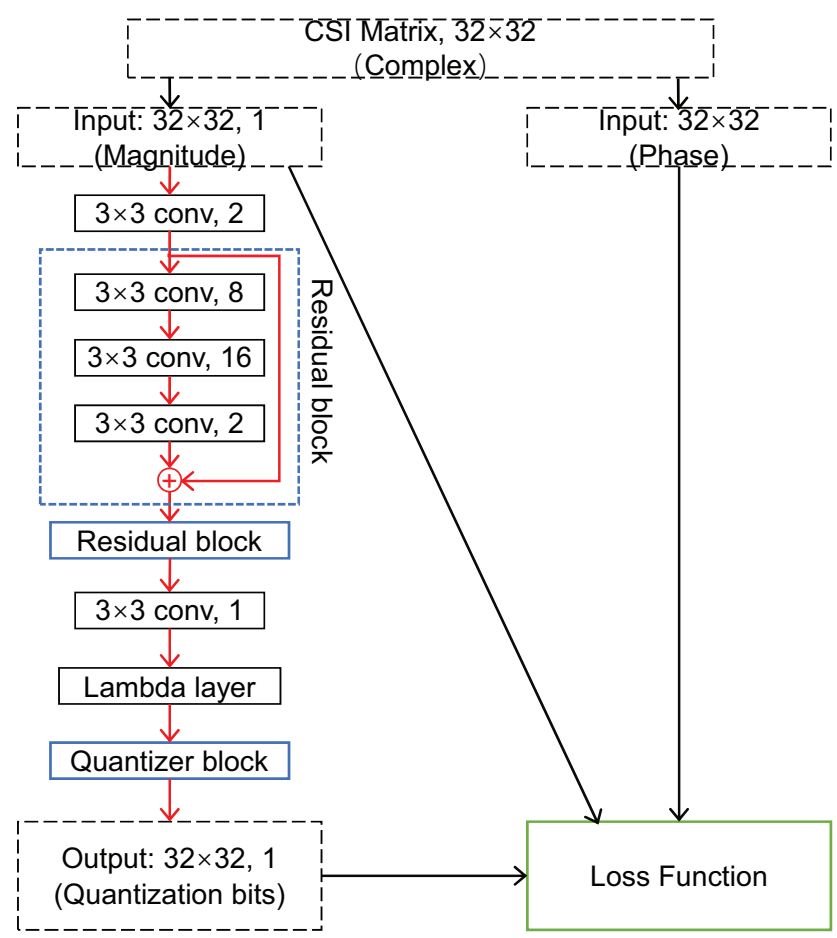

Fig. 6: Illustration of magnitude-adaptive phase quantization.

However, the heuristic method of [8] is inflexible with respect to the segments. Given different mean bitwidth constraints, we have to determine different allocation based on heuristics. Thus, we propose a more flexible and general design method in this work.

We propose an innovative DNN to solve the bit allocation problem. This new framework PhaseQuan can optimize the allocation of MAPQ quantization bits for phase based on unsupervised learning. As shown in Fig. 6, PhaseQuan utilizes the magnitudes as the input to DL network and includes CSI phases, magnitudes and corresponding quantization bits in its loss function. The input of magnitudes pass through a $3 \times 3$ convolutional layer and two residual block, which explore the potential spatial correlation within the CSI matrix. The ensuing convolutional layer, lambda layer, and quantizer block are used to project the output of the last hidden layer to quantization bits. The convolutional layer first utilizes the "sigmoid" activation to project the input within $[0,1]$. The lambda layer is defined as $\log _{2}\left(\frac{1}{x+\epsilon}\right)$, in which $\epsilon>0$ is a small value to ensure non-zero denominator. Here, $\frac{1}{x+\epsilon}$ corresponds to the number of quantization intervals. Logarithm $\log _{2}(\cdot)$ and the quantizer module can map the number of quantization intervals to the number of quantization bits. 
To minimize $\mathbf{E}_{(\mathbf{M}, \mathbf{P})}\left(\left\|\mathbf{M} \odot\left(e^{j \mathbf{P}}-e^{j \hat{\mathbf{P}}}\right)\right\|^{2}\right)$ within the constraint on the quantization bitwidth, we adopt the entropy of phase as the optimization regularizer as more quantization bits lead to higher phase entropy. Thus, we propose a loss function

$$
L(\mathbf{M}, \mathbf{P}, \mathbf{Y})=L_{m}(\mathbf{M}, \mathbf{P}, \mathbf{Y})+\lambda L_{y}(\mathbf{Y}),
$$

where $\mathrm{Y}$ is a matrix of integer elements representing the number of quantization bits for the corresponding CSI matrix element, optimized by the PhaseQuan. Since the quantization bits for each phase is variable, the codes are not uniquely decodable without knowing the quantization bits for each phase. By approximating $\mathrm{M}$ using $\hat{\mathbf{M}}$, the gNB can infer the corresponding bits of each phase from the recovered magnitude for uniquely decoding. Consequently, the phase quantization error evaluation function is set to be

$$
L_{m}(\mathbf{M}, \mathbf{P}, \mathbf{Y})=\mathbf{E}_{(\mathbf{M}, \mathbf{P})}\left(\left\|\hat{\mathbf{M}} \odot e^{j(\hat{\mathbf{P}}-\mathbf{P})}\right\|^{2}\right),
$$

in which $\hat{\mathbf{P}}$ is the quantized phase matrix. Additionally, define an entropy $H\left(\check{\mathbf{P}}_{i, j}\right)$ for quantized phase $\check{\mathbf{P}}_{i, j}$ that corresponds to magnitude $\mathbf{M}_{i, j}$. In this paper, we assume the phase to be uniformly distributed over $2 \pi$ [21]. Consequently, $H\left(\check{\mathbf{P}}_{i, j}\right)=\mathbf{Y}_{i, j}$. As a result, we use

$$
L_{y}(\mathbf{Y})=\mathbf{E}_{(\mathbf{M}, \mathbf{P})}\left(\frac{1}{Q_{f} \times N_{b}} \sum_{i, j} H\left(\check{\mathbf{P}}_{i, j}\right)\right)
$$

as an entropy regularizer to reduce number of quantization bits in phase feedback. Adjustable parameter $\lambda$ value governs the trade-off between the quantization bits and the reconstruction loss.

\section{Vi. Performance Evaluation}

\section{A. Experiment Setup}

We use the industry grade COST 2100 model [19] to generate massive MIMO channels for both training and testing of our DNN architecture. The training sample size is 70,000 and testing sample size is 30,000 . The values of epoch and batch size are set to 600 and 200, respectively. We test two scenarios:

(a) indoor channels with $5.1 \mathrm{GHz}$ uplink band and $5.3 \mathrm{GHz}$ downlink center frequency.

(b) semi-urban outdoor channels with $850 \mathrm{MHz}$ uplink band and $930 \mathrm{MHz}$ downlink center frequency. 
Uplink and downlink bandwidths of $20 \mathrm{MHz}$ and $5 \mathrm{MHz}$ are selected for the indoor and outdoor scenarios, respectively.

We place $\mathrm{gNB}$ at the center of a square area of lengths $20 \mathrm{~m}$ for indoor coverage and $400 \mathrm{~m}$ for outdoor coverage, respectively. We randomly position UEs within the coverage area. The gNB uses ULA with $N_{b}=32$ antennas and $N_{f}=1024$ subcarriers. After transforming the channel matrix $\mathbf{H}_{f}$ into the delay domain $\mathbf{H}_{t}$, only the first 32 rows are kept for feedback reporting due to sparsity.

To evaluate the accuracy of CSI recovery, we use normalized MSE

$$
\mathrm{NMSE}=\frac{1}{n} \sum_{k=1}^{n}\left\|\mathbf{H}_{d}^{k}-\hat{\mathbf{H}}_{d}^{k}\right\|^{2} /\left\|\mathbf{H}_{d}^{k}\right\|^{2},
$$

where $k$ and $n$ are the index and total number of samples in the testing set, respectively. We compare the CsiQnet and DualQnet with the CsiNet and DualNet-MAG for two quantization methods, respectively. The uniform quantization (UQ) and $\mu$-law non-uniform quantization are adopted $(\mu Q)$. For the $\mu$-law quantization, we use $\mu=255$ in the companding function

$$
F(x)=\operatorname{sgn}(x) \frac{\ln (1+\mu|x|)}{\ln (1+\mu)} .
$$

For the CsiQnet and CsiNet, CSI matrix is divided into two (real and imaginary) channels as the input to the DNN. We compare the CSI reconstruction performance under the compressed dimension $M=64$ and 256. For DualQnet and DualNet-MAG, we compare the CSI reconstruction performance under the compressed dimension $M=32$ and 128.

\section{B. CSI Reconstruction Performance Evaluation}

We compare the CSI reconstruction accuracy achieved from the above DL networks under five different bitwidths of 2,3,4,5 and 6. Single precision is used as the baseline for performance comparison. Fig. 7 shows the NMSE performance of our proposed CsiQnet and DualQnet.

As shown in Fig. 7, CsiQnet and DualQnet outperform CsiNet and DualNet-MAG using UQ and $\mu \mathrm{Q}$ obviously, and can achieve comparable performance with single precision data type using only 5 bits. As expected, non-uniform $\mu$-law quantization ( $\mu \mathrm{Q})$ outperforms uniform quantization (UQ) obviously, except for the extreme cases where the NMSE is greater than $10 \mathrm{~dB}$. For the CsiQnet, 2 bits quantization can achieve comparable performance as the $\mu \mathrm{Q}$ using 5 bits in indoor case and 6 bits in outdoor case. For DualQnet, 2 bits quantization can achieve even better performance than 6-bit $\mu \mathrm{Q}$. 


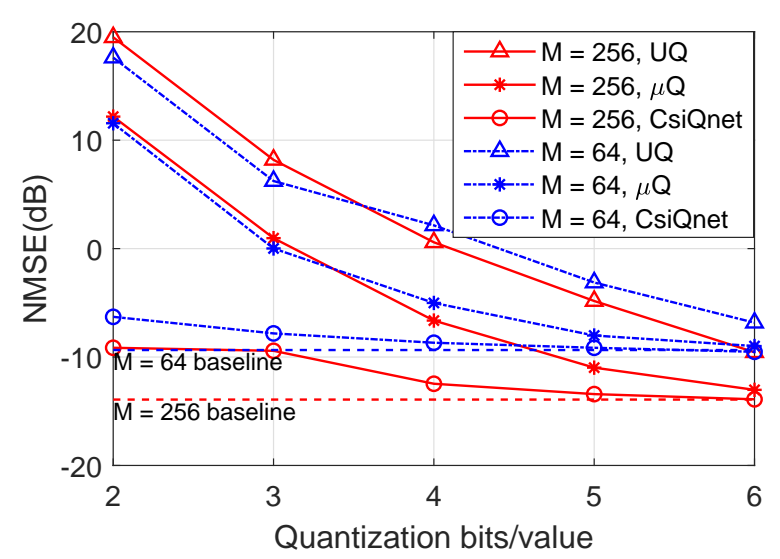

(a) CsiQnet indoor

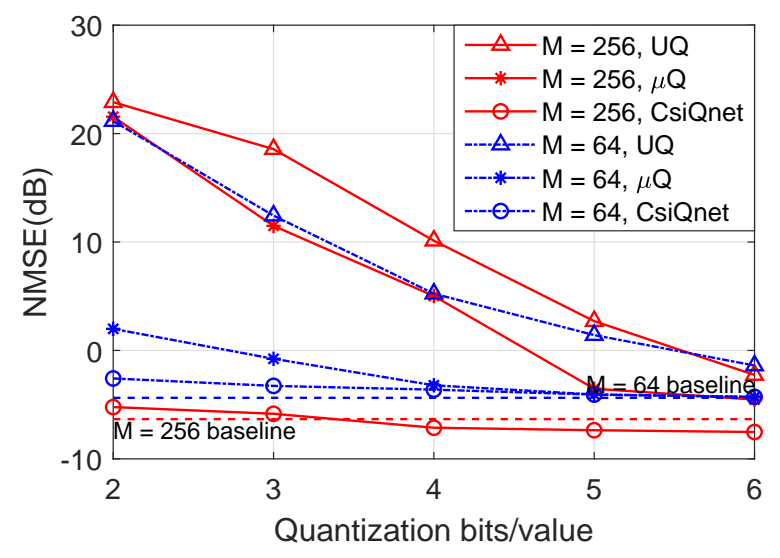

(c) CsiQnet outdoor

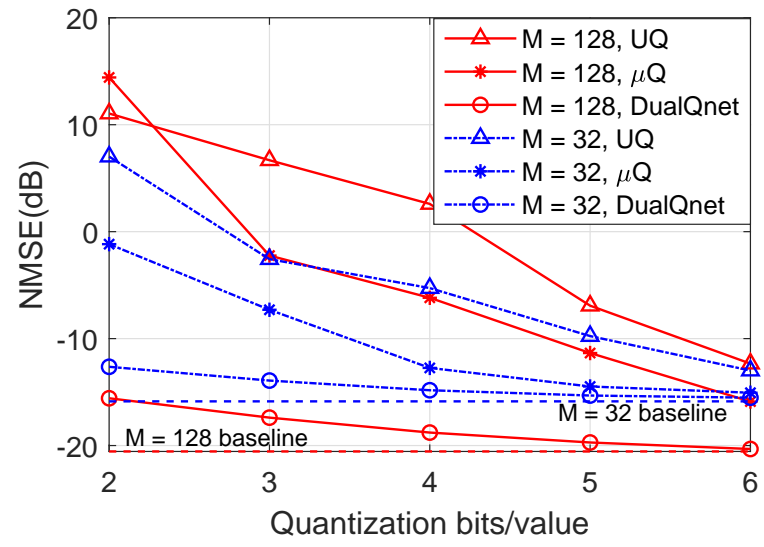

(b) DualQnet indoor

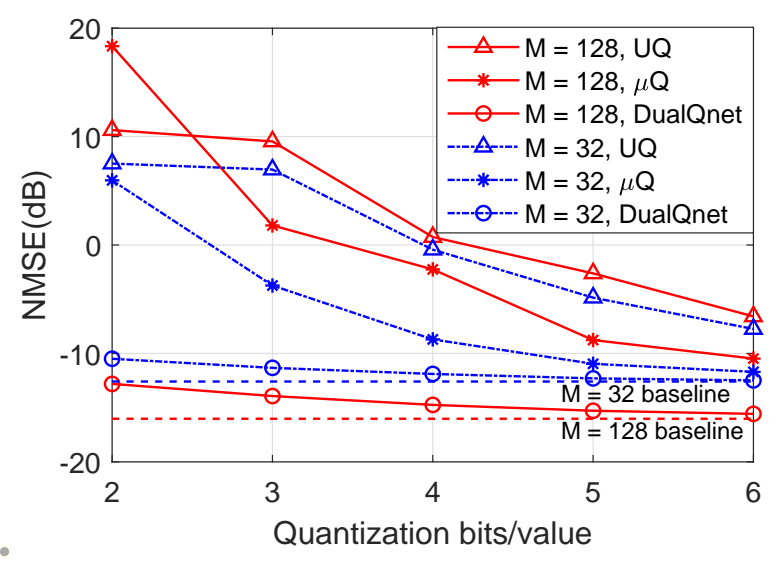

(d) DualQnet outdoor

Fig. 7: CSI recovery comparison at various quantization levels.

From the results in Fig. 7, we observe more robust performance when compressed dimension $M$ is relatively small. The NMSE degrades faster when $M$ is large. The possible reason is that the lower dimension compression relies on principal components, while high-accuracy reconstruction further requires more detailed information of compressed vectors. Thus, smaller feedback error can lead to a larger degradation in reconstruction accuracy when compression ratio is low. Interestingly, CsiQnet could outperform the baseline in outdoor scenario for $M=256$. One possible reason is that the quantized value can help DNN overcome some cases of local minima during training.

For a more comprehensive comparison, we further retrain the decoder networks of CsiNet and DualNet-MAG after quantizing the codewords from their encoder networks, and compare 


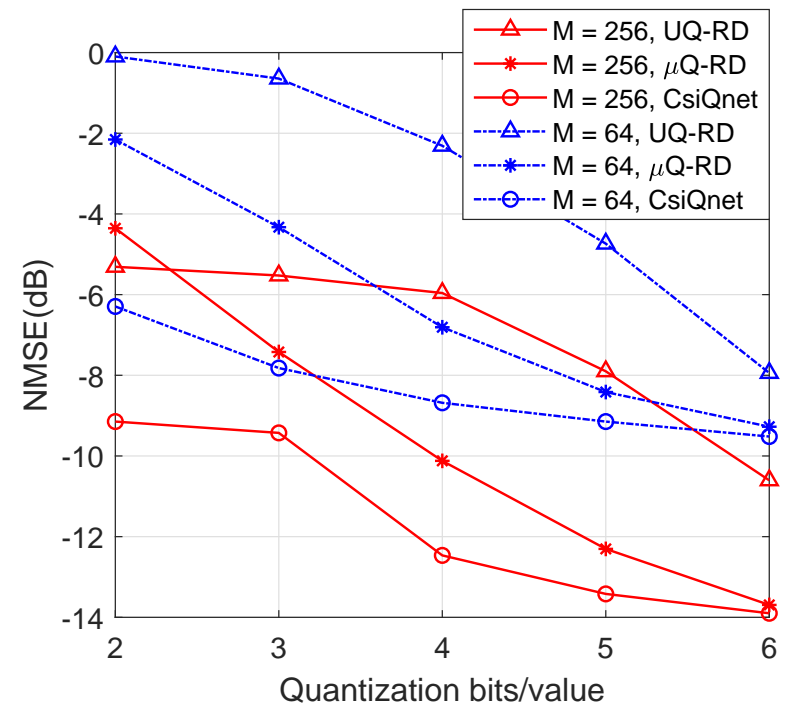

(a) CsiQnet indoor

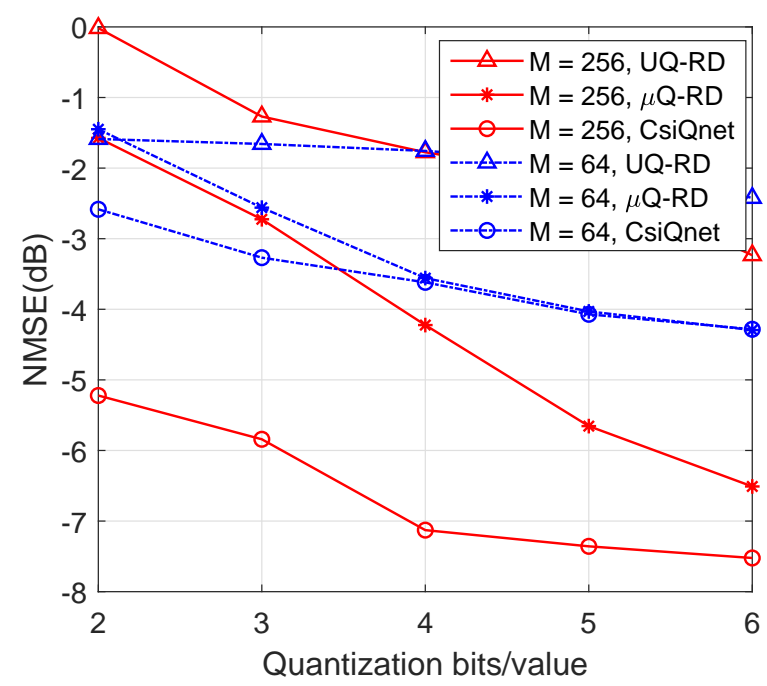

(c) CsiQnet outdoor

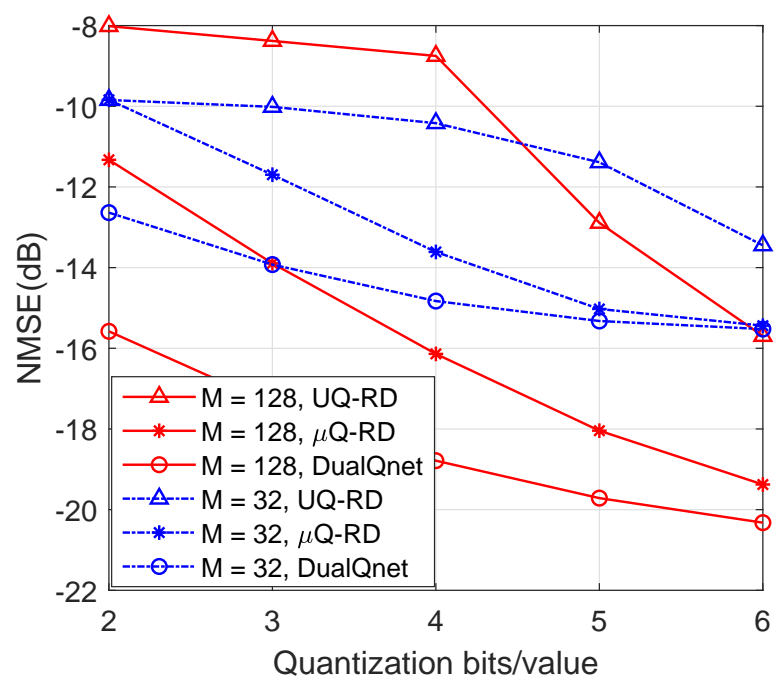

(b) DualQnet indoor

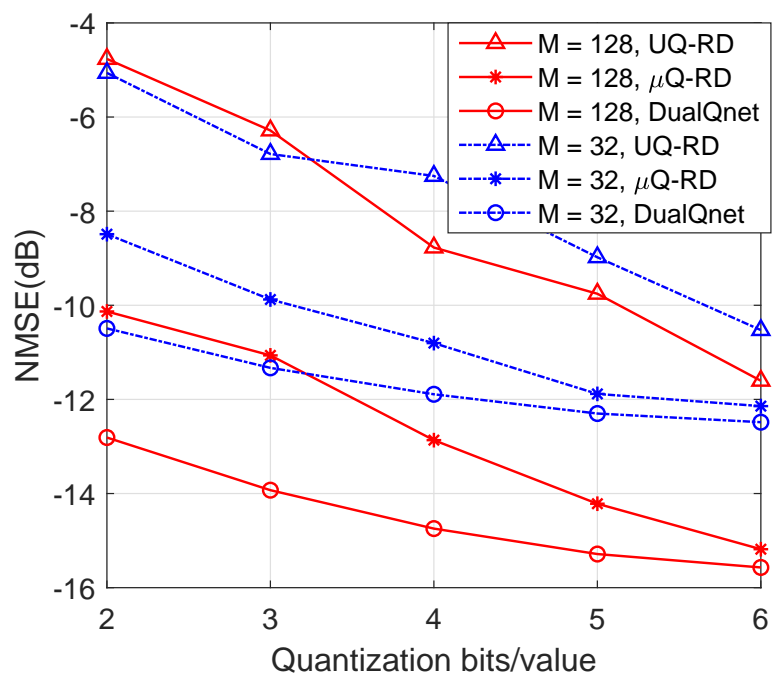

(d) DualQnet outdoor

Fig. 8: CSI recovery comparison at different quantization levels between CQNet and retrained decoder networks (RD).

their NMSE with CsiQnet and DualQnet. Both UQ and $\mu \mathrm{Q}$ are used to quantize the codewords from CsiNet and DualNet-MAG encoders. Denote the retrained decoder network results by RD, Fig. 8 shows the NMSE performance comparison of our proposed CsiQnet and DualQnet with CsiNet and DualNet-MAG when used with the retrained decoder. As shown in Fig. 8, CsiQnet and DualQnet both outperform CsiNet and DualNet-MAG under quantization and the 
TABLE I: Average required bits/value after entropy encoding when the quantization bits/value are 5 and 6 .

\begin{tabular}{c|c|c|c|c|c|c}
\hline Scenario & Network & Dimension & $\begin{array}{c}\mu \mathrm{Q} \\
5 \text { bits/value }\end{array}$ & $\begin{array}{c}\text { CQNet } \\
5 \text { bits/value }\end{array}$ & $\begin{array}{c}\mu \mathrm{Q} \\
6 \text { bits/value }\end{array}$ & $\begin{array}{c}\text { CQNet } \\
6 \text { bits/value }\end{array}$ \\
\hline \multirow{4}{*}{ Indoor } & \multirow{3}{*}{ CsiNet } & 64 & 4.36 & 3.88 & 5.37 & 4.41 \\
\cline { 3 - 7 } & \multirow{2}{*}{ DualNet } & 256 & 4.32 & 3.91 & 5.34 & 4.46 \\
\cline { 3 - 7 } & & 128 & 4.38 & 4.28 & 5.39 & 4.54 \\
\hline \multirow{3}{*}{ Outdoor } & \multirow{2}{*}{ CsiNet } & 64 & 4.37 & 3.71 & 5.38 & 4.15 \\
\cline { 3 - 7 } & \multirow{2}{*}{ DualNet } & 256 & 4.30 & 3.04 & 5.17 & 3.58 \\
\cline { 3 - 7 } & & 128 & 4.28 & 4.23 & 5.31 & 4.47 \\
\cline { 3 - 7 } & & 4.27 & 3.88 & 5.28 & 4.96 \\
\hline
\end{tabular}

retrained decoder. In fact, the accuracy gap increases with decreasing number of quantization bits/value. This result demonstrates that our end-to-end DNN framework jointly integrating CSI compression, quantization with reconstruction achieves better performance than combining individually optimized modules. Fig. 8 also shows that $\mu \mathrm{Q}$ generally delivers a better performance than UQ.

Once the CSI feedback is quantized, we can exploit entropy encoding to further compress the CSI feedback. Entropy encoding is a lossless scheme to compress digital data. We select the arithmetic coding [22], which is a simple and common entropy encoding to encode the quantized CSI coefficients. We consider $\mu \mathrm{Q}$ and CQNet with 5 and 6 bits per CSI value as examples. The average required numbers of bits per CSI value after entropy encoding are given in Table I] As shown in Table I, entropy encoding can help CQNet save additional 1 and 1.5 bits per CSI value on average when the quantization bits/value are 5 and 6 , respectively. It means that only 4 bits per CSI value are required by entropy encoding to deliver comparable performance previously achieved by float32 data type. We also find that, compared with $\mu \mathrm{Q}$, CQNet saves more bitwidth and achieves higher CSI reconstruction accuracy. In other words, although the entropy of CQNet is lower than that of $\mu \mathrm{Q}$, CQNet packs more useful information in its codewords for CSI reconstruction. 


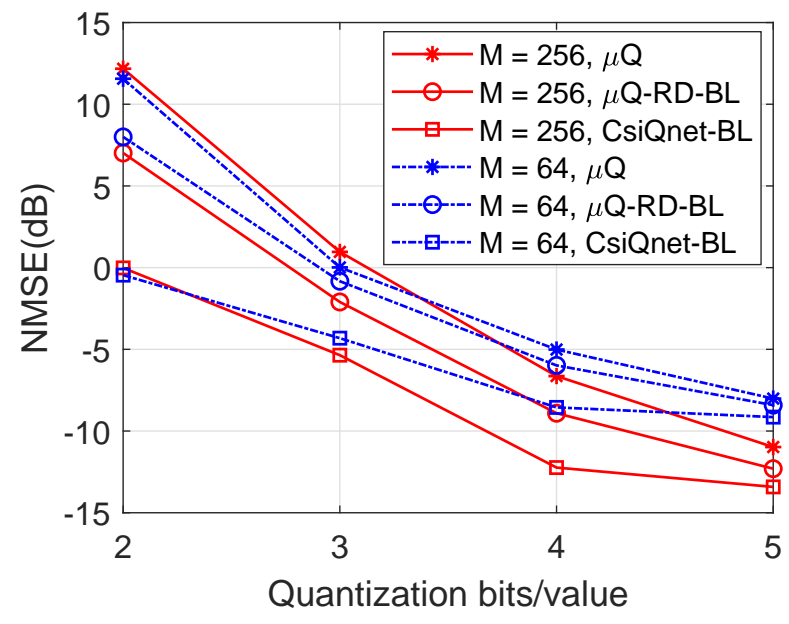

(a) CsiQnet indoor

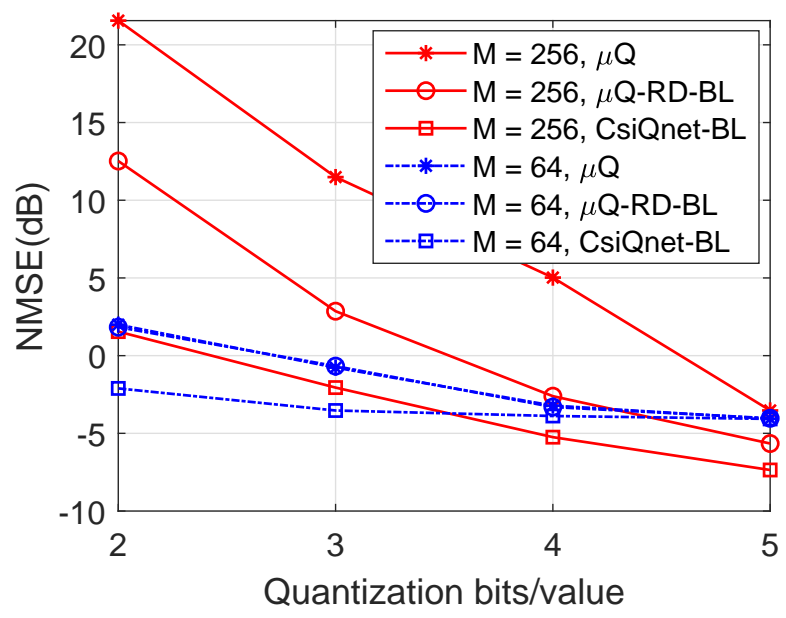

(c) CsiQnet outdoor

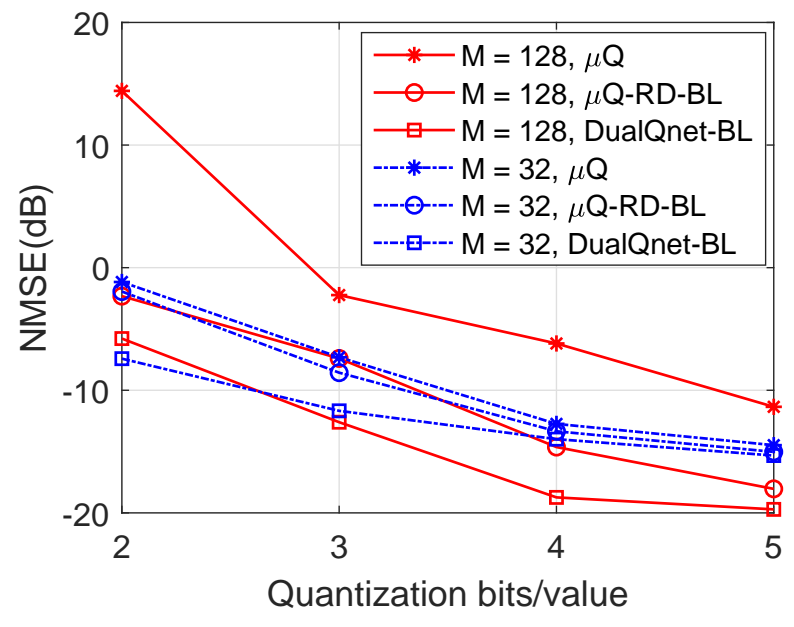

(b) DualQnet indoor

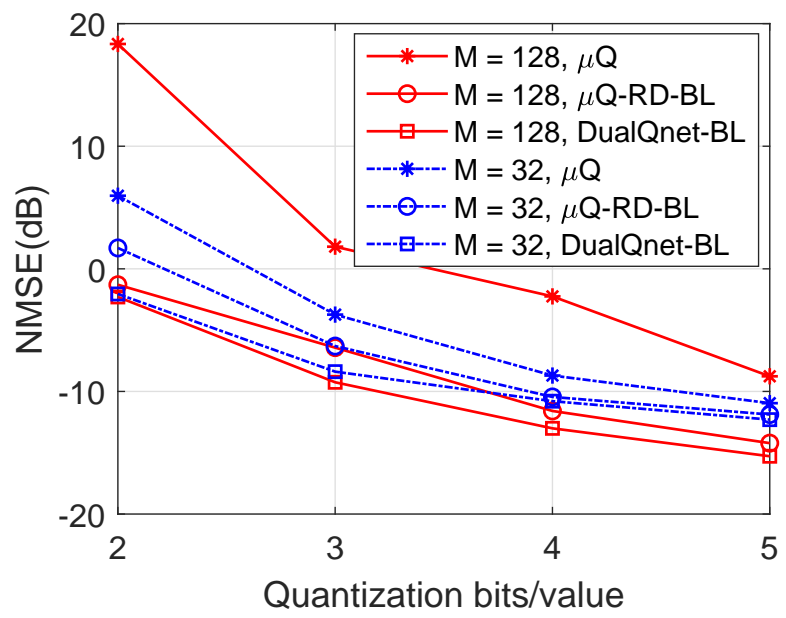

(d) DualQnet outdoor

Fig. 9: CSI recovery comparison at different quantization levels considering band limitation.

\section{Robustness Evaluation}

To further test the robustness of CsiQnet and DualQnet, we consider the case when there is only one neural network trained for the codewords quantization and reconstruction but different bitwidths may be required due to the bandwidth changes. Fig. 7 Thows that CsiQnet and DualQnet using 5 bits can achieve the similar performance as CsiNet and DualNet-MAG using single precision. As a result, we can select 5 quantization bits/value as an example to evaluate the robustness of CsiQnet and DualQnet. Since $\mu \mathrm{Q}$ outperforms UQ clearly when quantization bits/value is 5, we select $\mu \mathrm{Q}$ and $\mu \mathrm{Q}-\mathrm{RD}$ for CsiNet and DualNet-MAG as a reference to test 
the robustness.

Considering a DNN already trained for the 5 quantization bits/value. If the UE does not have sufficient bandwidth to transmit each codeword using 5 bits, we need to further shorten the codewords using fewer bits: 2, 3 and 4 bits/value. Fig. 9 shows the performance of CsiQnet and DualQnet under different bandwidth limitations (BL). As shown in Fig. 9, the performance of CsiQnet and DualQnet in BL cases using fewer bits for the quantized codewords clearly outperforms the CsiNet and DualNet-MAG using $\mu \mathrm{Q}$ and $\mu \mathrm{Q}-\mathrm{RD}$. On the other hand, although CsiQnet and DualQnet in BL cases are more robust than other methods, their performance has a relatively obvious degradation when the quantization bits/value falls below 4. In our future work, we plan to extend the CsiQnet and DualQnet to variable quantization bitwidth.

\section{Quantization Evaluation}

In this subsection, we analyze the performance of our quantizer module, and try to go inside the DL networks to explore why CsiQnet and DualQnet outperform the UQ and $\mu \mathrm{Q}$ methods.

We first compare the performance of our approximated rounding function with the actual rounding function, and show the MSE between the output of $\widetilde{\operatorname{Rnd}}(\cdot)$ and round function in Numpy. Table II shows the MSE under different number of quantizations bits. As shown in Table III. MSE generally is near $-24 \mathrm{~dB}$, which is negligible compared with the rounded integer. We also compare the NMSE of the CSI reconstruction between using $\widetilde{\operatorname{Rnd}}(\cdot)$ and $\operatorname{Rnd}(\cdot)$. The differences are less than $-0.15 \mathrm{~dB}$, which means the approximate $\widetilde{\operatorname{Rnd}}(\cdot)$ works well for the quantization.

It would be helpful for us to examine the effect of quantization error empirically to understand the performance of different CSI feedback methods under study.

We measure the normalized mean square quantization error (NMSQE) defined as $\mathbf{E}\left[\frac{\|\mathbf{s}-\hat{\mathbf{s}}\|^{2}}{\|\mathbf{s}\|^{2}}\right]$. We choose 5 quantization bits. We select CsiNet with $M=256$ and DualNet-MAG with $M=128$ to demonstrate the quantization error of UQ and $\mu \mathrm{Q}$. As shown in Table III, NMSQE of $\mu \mathrm{Q}$ is obviously lower than the corresponding NMSQE from UQ for both CsiNet and DualNet. The comparison clearly demonstrates one reason why $\mu \mathrm{Q}$ can provide better CSI recovery accuracy than UQ, as shown in our results in this section. 
TABLE II: MSE(dB) of the approximated rounding function

\begin{tabular}{c|c|c|c|c|c}
\hline & & \multicolumn{2}{|c|}{ Indoor } & \multicolumn{2}{c}{ Outdoor } \\
\hline \multirow{5}{*}{ CsiQnet } & Bits & $M=64$ & $M=256$ & $M=64$ & $M=256$ \\
\cline { 2 - 6 } & 2 & -24.4319 & -24.4578 & -21.7272 & -24.4584 \\
\cline { 2 - 6 } & 3 & -24.2357 & -24.1985 & -23.9548 & -24.2606 \\
\cline { 2 - 6 } & 4 & -24.1401 & -24.1621 & -24.0639 & -24.1691 \\
\cline { 2 - 6 } & 5 & -24.1564 & -24.1512 & -24.1445 & -24.1482 \\
\cline { 2 - 6 } & 6 & -24.0967 & -24.1336 & -24.1289 & -24.1554 \\
\hline \multirow{5}{*}{ DualQnet } & Bits & $M=32$ & $M=128$ & $M=32$ & $M=128$ \\
\cline { 2 - 6 } & 2 & -24.3763 & -24.9159 & -24.1652 & -24.4255 \\
\cline { 2 - 6 } & 3 & -24.2162 & -24.2249 & -24.1463 & -24.2362 \\
\cline { 2 - 6 } & 4 & -24.2008 & -24.1677 & -24.1937 & -24.1402 \\
\cline { 2 - 6 } & 5 & -24.1409 & -24.1249 & -24.2236 & -24.1325 \\
\cline { 2 - 6 } & 6 & -24.1286 & -24.1522 & -24.0995 & -24.131 \\
\hline
\end{tabular}

TABLE III: NMSQE(dB) performance of UQ and $\mu \mathrm{Q}$.

\begin{tabular}{l|c|c}
\hline & Indoor & Outdoor \\
\hline CsiNet-UQ, $M=256$ & -11.1237 & -12.7929 \\
\hline CsiNet- $\mu \mathrm{Q}, M=256$ & -19.3166 & -21.5008 \\
\hline DualNet-UQ, $M=128$ & -11.33 & -7.58515 \\
\hline DualNet- $\mu \mathrm{Q}, M=128$ & -19.2605 & -19.5497 \\
\hline
\end{tabular}

\section{E. Phase Quantization}

In order to flexibly allocate the phase quantization bits under different bandwidth limitation and reconstruction accuracy requirement, we train PhaseQuan under different $\lambda$ values and illustrate the effect of $\lambda$ on average phase quantization bits and reconstruction accuracy. Intuitively, smaller $\lambda$ leads to higher reconstruction accuracy, though at a cost of higher feedback overhead. The converse also holds.

The bitwidth-NMSE trade-off under different $\lambda$ is shown in Fig. 10, We use the phase quantization method in DualNet-MAG as the baseline, and evaluate 3 cases of magnitude knowledge for CSI reconstruction. We consider (a) perfect CSI magnitude; (b) DualQnet after dimension compression using 5 quantization bits with $M=128$, and (c) DualQnet using 5 quantization bits with $M=32$. As shown in Fig. 10, the performances of PhaseQuan are comparable to the baseline with better flexibility by adjusting $\lambda$. NMSE decreases with increasing 


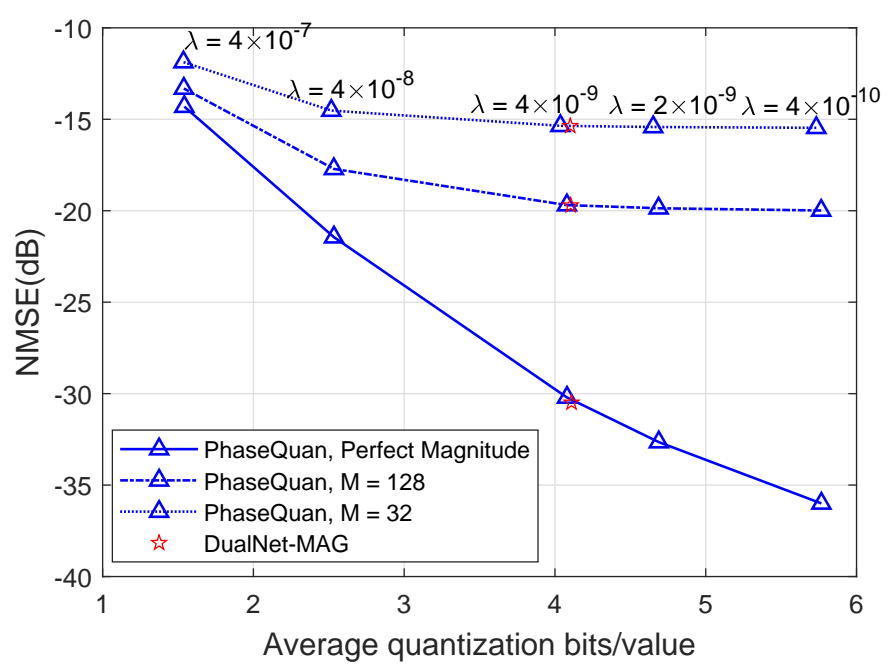

(a) Indoor

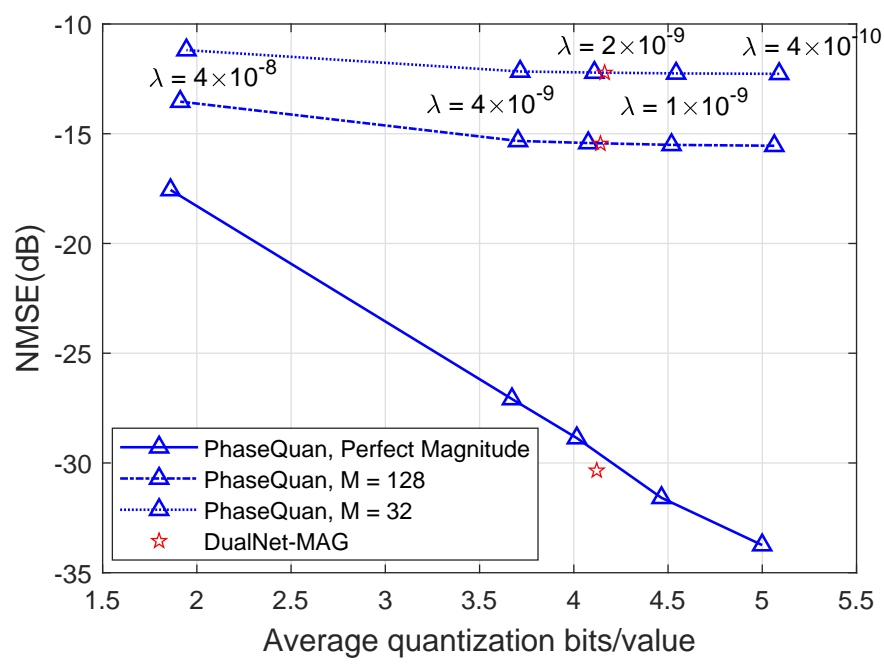

(b) Outdoor

Fig. 10: Quantization bits-NMSE trade-off under different $\lambda$.

quantization bitwidth. With a large $\lambda$ value, the DNN tries to reduce the number of quantization bits, which in turn degrades NMSE. To find the suitable $\lambda$ for a given NMSE, we can first select several candidate values of $\lambda$ to train the PhaseQuan as reference anchors. By interpolating $\lambda$ according to users requirements in terms of CSI reconstruction accuracy and the available feedback bandwidth, we can obtain the phase quantization parameters under these constraints.

On the other hand, with lower accuracy in magnitude, the influence of quantization bits becomes weaker. This means that we can save bits in phase quantization according to the 
magnitude accuracy. For example, in the indoor cases when $M=32$, phase quantization using 2.5 bits/value and 4.1 bits/value can generate similar NMSE. In the outdoor cases when $M=32$, phase quantization using 1.9 bits/value and 4.1 bits/value can generate similar NMSE.

In future works, we should jointly optimize the compression and encoding of magnitude and phase.

\section{CONCLUSIONS}

The previous success of DL in achieving more efficient CSI feedback for massive MIMO systems in FDD deployment strongly motivates investigation of bandwidth efficient encoding of the compressed CSI coefficients. In this paper, we propose a comprehensive DL-based CSI feedback framework CQNet to jointly optimize the dimension compression, codewords quantization, and recovery of CSI matrices for massive MIMO transmission. We integrate CQNet with two DL-based CSI feedback mechanisms, and demonstrate clear feedback savings while maintaining downlink CSI reconstruction accuracy at the massive MIMO base station. CQNet significantly outperforms uniform quantization and $\mu$-law quantization, and can reduce CSI encoding from 32 to 5 bits with little loss of CSI reconstruction accuracy. We achieve additional feedback reduction by introducing additional entropy encoding. We further present a DL-based phase quantizer for CSI feedback framework DualNet-MAG that exploits bi-directional correlation and improve the flexibility to manage the trade-off between CSI reconstruction accuracy and feedback bandwidth.

\section{REFERENCES}

[1] E. G. Larsson, O. Edfors, F. Tufvesson, and T. L. Marzetta, "Massive MIMO for next generation wireless systems," IEEE Commun. Mag, vol. 52, no. 2, pp. 186-195, February 2014.

[2] X. Rao and V. K. N. Lau, "Distributed compressive CSIT estimation and feedback for FDD multi-user massive MIMO systems," IEEE Trans. Signal Process., vol. 62, no. 12, pp. 3261-3271, June 2014.

[3] Z. Lv and Y. Li, "A channel state information feedback algorithm for massive MIMO systems," IEEE Commun. Lett, vol. 20, no. 7, pp. 1461-1464, July 2016.

[4] Z. Gao, L. Dai, Z. Wang, and S. Chen, "Spatially common sparsity based adaptive channel estimation and feedback for FDD massive MIMO,” vol. 63, no. 23, pp. 6169-6183, Dec 2015.

[5] H. Son and Y. Cho, "Analysis of compressed CSI feedback in MISO systems," IEEE Wireless Commun. Lett, pp. 1-1, 2019.

[6] H. He, C. Wen, S. Jin, and G. Y. Li, "Deep learning-based channel estimation for beamspace mmwave massive MIMO systems," IEEE Wireless Commun. Lett, vol. 7, no. 5, pp. 852-855, Oct 2018.

[7] C. Wen, W. Shih, and S. Jin, "Deep learning for massive MIMO CSI feedback," IEEE Wireless Commun. Lett., vol. 7 , no. 5, pp. 748-751, Oct 2018. 
[8] Z. Liu, L. Zhang, and Z. Ding, "Exploiting bi-directional channel reciprocity in deep learning for low rate massive MIMO CSI feedback," IEEE Wireless Commun. Lett, vol. 8, no. 3, pp. 889-892, June 2019.

[9] L. Theis, W. Shi, A. Cunningham, and F. Huszár, in Lossy image compression with compressive autoencoders, April 2017.

[10] “IEEE standard for floating-point arithmetic," IEEE Std 754-2008, pp. 1-70, Aug 2008.

[11] J. Johnson, “Rethinking floating point for deep learning," arXiv preprint arXiv:1811.01721, 2018.

[12] T. Wang, C. Wen, S. Jin, and G. Y. Li, "Deep learning-based CSI feedback approach for time-varying massive MIMO channels," IEEE Wireless Commun. Lett, vol. 8, no. 2, pp. 416-419, April 2019.

[13] C. Lu, W. Xu, H. Shen, J. Zhu, and K. Wang, "MIMO channel information feedback using deep recurrent network," IEEE Commun. Lett, vol. 23, no. 1, pp. 188-191, Jan 2019.

[14] S. Hochreiter and J. Schmidhuber, "Long short-term memory," Neural computation, vol. 9, no. 8, pp. $1735-1780,1997$.

[15] N. Wang, J. Choi, D. Brand, C. Chen, and K. Gopalakrishnan, "Training deep neural networks with 8-bit floating point numbers," in Advances in neural information processing systems, 2018, pp. 7675-7684.

[16] J. Guo, C. Wen, S. Jin, and G. Y. Li, "Convolutional neural network based multiple-rate compressive sensing for massive MIMO CSI feedback: Design, simulation, and analysis," arXiv preprint arXiv:1906.06007, 2019.

[17] C. Lu, W. Xu, S. Jin, and K. Wang, "Bit-level optimized neural network for multi-antenna channel quantization," IEEE Wireless Commun. Lett, pp. 1-1, 2019.

[18] R. H. Jr and A. Lozano, Foundations of MIMO communication. Cambridge University Press, 2018.

[19] L. Liu, C. Oestges, J. Poutanen, K. Haneda, P. Vainikainen, F. Quitin, F. Tufvesson, and P. D. Doncker, “The COST 2100 MIMO channel model," IEEE Wireless Commun., vol. 19, no. 6, pp. 92-99, December 2012.

[20] P. Belotti, C. Kirches, S. Leyffer, J. Linderoth, J. Luedtke, and A. Mahajan, "Mixed-integer nonlinear optimization,” Acta Numerica, vol. 22, pp. 1-131, 2013.

[21] P. . Kildal and K. Rosengren, "Correlation and capacity of MIMO systems and mutual coupling, radiation efficiency, and diversity gain of their antennas: simulations and measurements in a reverberation chamber," IEEE Commun. Mag, vol. 42, no. 12, pp. 104-112, Dec 2004.

[22] I. Witten, R. Neal, and J. Cleary, "Arithmetic coding for data compression," Communications of the ACM, vol. 30, no. 6, pp. 520-540, 1987. 